LA-UR-02-4901

Approved for public release;

distribution is unlimited.

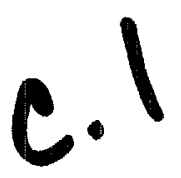

Title:
Submitted to: Solar Wind 10 Conference Proceedings

CMES AT HIGH NORTHERN LATITUDES DURING SOLAR MAXIMUM: ULYSSES AND SOHO CORRELATED OBSERVATIONS

Author(s): $\begin{aligned} & \text { Daniel B. Reisenfeld, Los Alamos National Laboratory } \\ & \text { (see attached sheet for additional authors) }\end{aligned}$

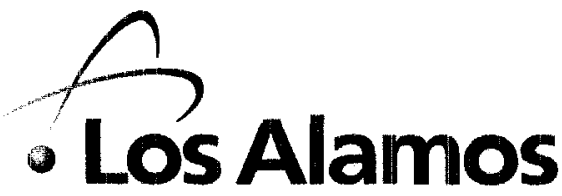

NATIONAL LABORATORY

Los Alamos National Laboratory, an affirmative action/equal opportunity employer, is operatenny the University of California for the U.S. Department of Energy under contract W-7405-ENG-36. By acceptance of this article, the publisher recognizes that the U.S. Government retains a nonexclusive, royalty-free license to publish or reproduce the published form of this contribution, or to allow others to do so, for U.S. Government purposes. Los Alamos National Laboratory requests that the publisher identify this article as work performed under the auspices of the U.S. Department of Energy. Los Alamos National Laboratory strongly supports academic freedom and a researcher's right to publish; as an institution, however, the Laboratory does not endorse the viewpoint of a publication or guarantee its technical correctness. 


\title{
CMEs at High Northern Latitudes During Solar Maximum: Ulysses and SOHO Correlated Observations
}

\author{
Daniel B. Reisenfeld", John T. Gosling", John T. Steinberg*, Pete Riley ${ }^{\dagger}$, \\ Robert J. Forsyth" and O. Chris St. Cyr ${ }^{\ddagger}$ \\ "Space and Atmospheric Sciences, MS-D466, Los Alamos National Laboratory, Los Alamos, NM 87544 \\ ${ }^{t}$ SAIC, 10260 Campus Point Drive, San Diego, CA 92121 \\ The Blacklett Laboratory, Imperial College, London, UK \\ ${ }^{\ddagger}$ Catholic University of America, Code 682, NASA-Goddard Space Flight Center, Greenbelt, MD 20771
}

\begin{abstract}
From September through November 2001, Ulysses was almost continuously immersed in polar coronal hole (CH) flow during its northern polar pass of the Sun. For much of this time, the flow was fast $(>700 \mathrm{~km} / \mathrm{s})$ and steady, quite similar to the steady unstructured flow observed during Ulysses' first polar orbit near solar minimum. During the three months Ulysses transited the northern polar $\mathrm{CH}$ it observed 5 coronal mass ejections (CMEs). Of these, two were clearly over-expanding and two were at least partially driven by overexpansion. The phenomenon of over-expansion was frequently observed at high latitudes during Ulysses' first orbit. The recurrence of over-expanding CMEs during the second orbit at high latitudes indicates that this is a phenomenon apparently unique to and typical of CMEs embedded in polar CH flow. Ulysses was nearly above the solar limb during this three-month interval, providing an opportunity to use LASCO/SOHO observations to study the initial velocity profiles of the CMEs observed further out by Ulysses. These initial conditions were used as inputs into a hydrodynamic code, the results of which are reported here.
\end{abstract}

\section{INTRODUCTION}

From September through November 2001, Ulysses was almost continuously immersed in polar coronal hole $(\mathrm{CH})$ flow as it transited the northern polar regions of the Sun. This polar pass coincided with the phase of the solar cycle immediately following solar maximum when a new polar $\mathrm{CH}$ had formed over the North Pole [1]. This $\mathrm{CH}$ was much smaller in extent than that observed during the first orbit: during the first orbit, near solar minimum, Ulysses observed continuous $\mathrm{CH}$ flow at all latitudes above $\sim 35^{\circ}$, whereas Ulysses observed the recent $\mathrm{CH}$ only above latitudes of $70^{\circ} \mathrm{N}$. Although covering a much narrower range of latitudes, the properties of this flow were nearly identical to those of the first orbit: a fast, steady wind speed, a low density, and a high temperature. The one notable difference is that whereas during the first pair of polar passes, there were very few coronal mass ejections (CMEs), this was not the case here. During the first orbit, Ulysses encountered only 6 CMEs over the course of $\sim 3$ years of immersion in polar $\mathrm{CH}$ flow (1-1/2 years each in the southern and northern $\mathrm{CHs}$, respectively). However, in the brief three-month period considered here, it intercepted 5 CMEs. Of these, 2 were clearly overexpanding (expansion driven by an internal overpressure [2]) and 2 were at least partially driven by over-expansion. Because these 5 CMEs were embedded in otherwise steady flow, we were afforded an excellent opportunity to investigate CME evolution through interplanetary space without interference from other solar wind perturbations. This was an ideal situation for comparison to the hydrodynamic (HD) simulation of CME evolution. Additionally, because Ulysses was positioned almost directly above the Sun's North limb for this time period, the Ulysses observations could be correlated with remote sensing observations of CMEs made by instruments on board the Solar and Heliographic Observatory (SOHO).

We were able to identify the solar source of 3 of the 5 CMEs observed by Ulysses using LASCO and EIT images. We have used the LASCO observations to determine the initial speed profiles of these CMEs, 
and then used these as inputs into a 1-D HD model of $\mathrm{CME}$ evolution. Here, we describe the SOHO and Ulysses observations of 2 of these CMEs and compare the Ulysses observations to the simulation results. We then discuss the successes and pitfalls of such comparisons. This analysis is particularly relevant in view of the upcoming STEREO mission, which has the specific goal of investigating CME evolution via multi-spacecraft remote imaging and in situ observations.

\section{SOHO AND ULYSSES OBSERVATIONS}

Of the 5 CMEs, we present here the observational details of 2. Plasma observations were made by the Ulysses ion and electron spectrometers [3]. Solar coronagraphic images were obtained from the Large Angle Spectrometric Coronagraph (LASCO) experiment on SOHO [4]. Where possible, we make use of full-disk images obtained from the extreme ultraviolet imaging telescope (EIT) on SOHO [5] to locate the activity source leading to the $\mathrm{CME}$.

\section{The October 24, 2001 Event}

The first event occurred at the Sun on October 24, 2001 at 23:06 UT, appearing in LASCO as a partial halo at the North Pole. The event was clearly accelerating. When it first appeared in the $\mathrm{C} 3$ white light optical system, the projected velocity toward Ulysses was only about $100 \mathrm{kms}^{-1}$, but by the time it left the LASCO field-of-view (FOV) (at 28 solar radii, or $0.13 \mathrm{AU}$ ) it was moving at an almost constant velocity of $700 \mathrm{~km} / \mathrm{s}$. That this event is directed toward Ulysses is supported by EIT $284 \AA$ images that show the disappearance of an arcade having a northern boundary coincident with that of the North Pole $\mathrm{CH}$.

We have associated these SOHO observations with an event observed by Ulysses on 29 October - 1 November. The CME, shown in Figure 1, exhibited nearly classic over-expansion [2]. We identify this as a CME by the presence of counter-streaming electrons, a descending speed profile, an enhanced magnetic field, and smooth field rotation (not shown), which indicates a magnetic flux rope. The central portion of the CME showed a pressure minimum, sandwiched between two pressure pulses traveling away from the center. At this distance from the Sun, only the reverse compression had steepened into a shock. The CME was immersed in very steady flow, with a central speed of $750 \mathrm{~km} \mathrm{~s}^{-1}$, comparable to the speeds before and after the disturbance. Its dynamical evolution was therefore driven solely by the CME's internal overpressure. We also note that the central speed of 750 $\mathrm{km} \mathrm{s}^{-1}$ was very close to the leading edge speed of 700 $\mathrm{km} \mathrm{s}^{-1}$ observed at the limit of the LASCO FOV.

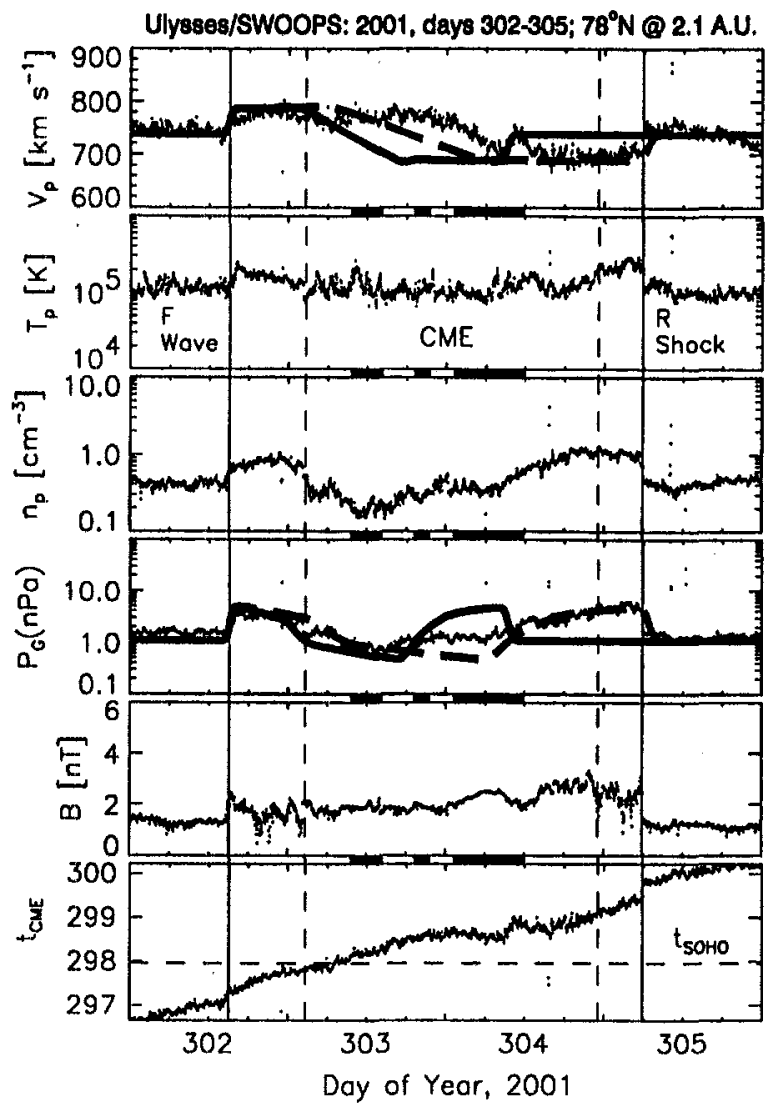

FIGURE 1. Ulysses plasma and magnetic field data for the CME associated with the 24 October event. The CME (between the dashed vertical lines) is bounded by a forward wave and a reverse shock. The panels show the solar wind proton flow speed $v_{p}$, proton temperature $T_{p}$, proton density $n_{p}$, total gas pressure (electrons + ions) $P_{G}$, magnetic field strength $B$, and the predicted time when the plasma departed the Sun $t_{\mathrm{CME}}$ (see text). The bottom panel also shows the

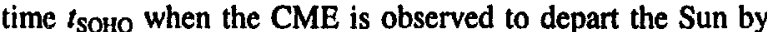
SOHO. The dark bands between panels show times of electron counterstreaming. In the panels for $v_{p}$ and $P_{G}$, the solid lines show the results from the 1-D HD simulation of the event, and the dashed lines show the simulation results scaled in time to match the duration of the observed CME.

The bottom panel of Figure 1 shows the time $t_{\mathrm{CMB}}$ that the CME plasma is predicted to have left the Sun: $t_{\mathrm{CME}}=t_{U}-r_{U} / v_{U}$, where $t_{U}, r_{U}$, and $v_{U}$ are the time of observation, heliocentric distance, and flow speed at Ulysses [6]. The time $t_{\mathrm{CME}}$ can be compared to the time $t_{\text {sOHo }}$ when the leading edge of the associated CME was first observed at the Sun by LASCO. The equation for $t_{\mathrm{CME}}$ assumes ballistic flow from the Sun, that is, no net acceleration or deceleration of the $\mathrm{CME}$ 
throughout its transit. The bottom panel of Figure 1 shows $t_{\mathrm{CME}}$, and the horizontal dashed line represents the time $t_{\text {SOHO }}$ (23:06 UT on 24 October) of the associated LASCO event. The time $t_{\text {SOHо }}$ preceded $t_{\text {CME }}$ for most of the CME, indicating that the plasma must have accelerated after initiation, which in fact is just what was observed in the LASCO images.

\section{The November 22, 2001 Event}

A large halo CME was observed by LASCO on 22 November associated with a pair of M-class flares, at 20:58 and 23:30 UT, respectively. The disk activity was observed by EIT in the SW quadrant of the Sun; thus it is not obvious that this event headed toward Ulysses. Nevertheless, by tracking the leading edge, we find that this event was moving extremely fast, with a projected speed of $1390 \mathrm{~km} \mathrm{~s}^{-1}$ toward Ulysses.

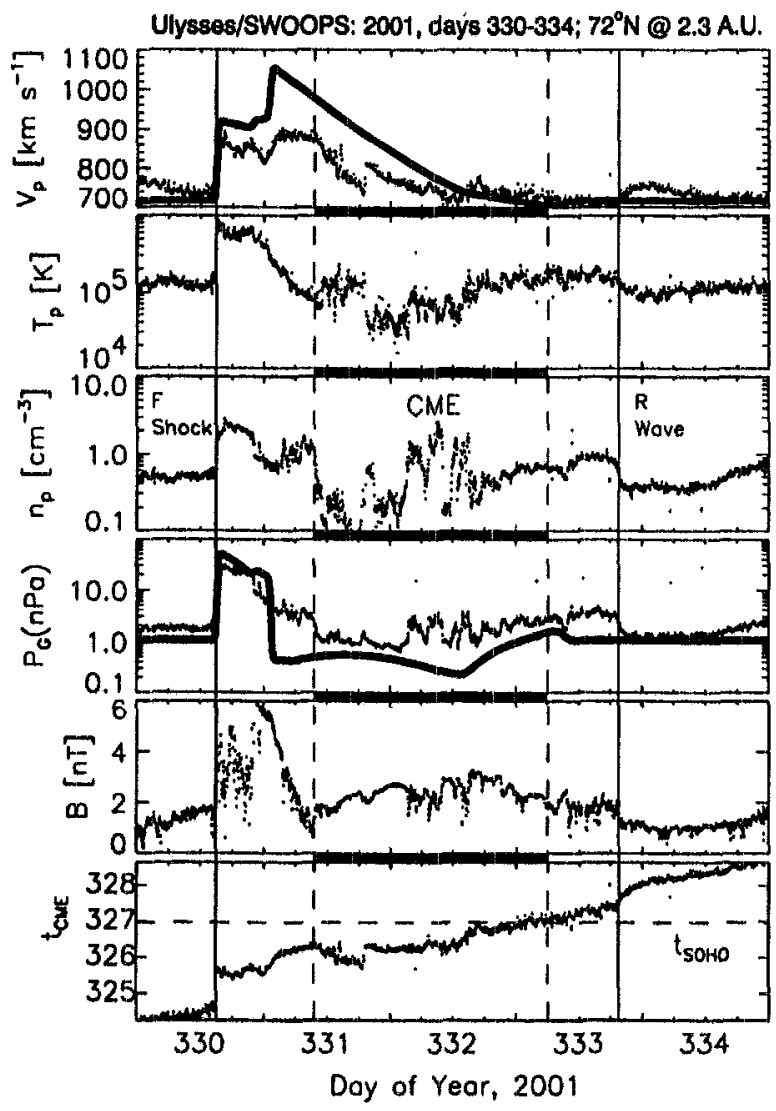

FIGURE 2. Ulysses plasma and field data for the CME associated with the LASCO 22 November event. The CME (between the dashed vertical lines) is bounded by a forward shock and a reverse wave. See Fig. 1 for explanation.

Figure 2 shows Ulysses plasma and field data at 2.3 AU for 26-29 November, which we have associated with the 22 November solar event. The event was remarkably strong, exhibiting counter-streaming; a forward shock at $890 \mathrm{~km} \mathrm{~s}^{-1}$ having a strength of 4.0 , and a central temperature depression. The CME had a strong magnetic field enhancement and a low field variance. The center showed a weak pressure minimum, and the event ended with a reverse wave propagating into the wind behind it. Thus, whereas the front half of the event was typical of a fast CME plowing into slower wind ahead, the rear half of the event had the shape of an over-expanding CME. The reverse wave was weak since it was propagating into receding plasma. The bottom panel of Figure 2 shows that $t_{\mathrm{CME}}$ preceded $t_{\text {Sоно }}$ throughout the event, implying there must have been significant deceleration in the heliosphere.

We believe that the Ulysses and SOHO observations correspond to the same event because (1) the Ulysses event was very energetic and no LASCO events which are likely to give rise to such a powerful event at Ulysses were observed at a reasonable time before or after the described event, and (2) an event at $A C E$ is observed on 24-26 November (not shown) which has a very similar magnetic profile to the Ulysses event, and for which the calculated values of $t_{\mathrm{CME}}$ lead to an obvious association with the LASCO event. Furthermore, 2-D and 3-D HD simulations by Riley et al. [7] and Odstrcil \& Pizzo [8], show that CME disturbances can undergo significant lateral expansion in the heliosphere; thus it is reasonable to expect that an event initially spanning a relatively localized angular extent near the Sun may span an angular region many times greater by the time it reaches heliocentric distances of order 1 AU.

\section{SIMULATIONS}

We have performed 1-D HD simulations to understand better the evolution of the above events through the heliosphere. Simulations of this sort have been performed in the past to model the evolution of the solar wind in general and CMEs in particular as they propagate through the heliosphere, but here we use SOHO observations to constrain partially the simulations by providing initial start times and speeds.

We use a simple 1-D HD simulation that reproduces the basic physics behind these events. The code predicts too strong an interaction between the CME and the ambient solar wind because it does not incorporate shear flows, which relieve pressure stresses. The code also neglects magnetic forces. Despite these limitations, our simulations have produced event profiles that are qualitatively, and 
sometimes quantitatively, similar to the disturbances observed far from the Sun.

All simulations were performed using a versatile numerical code (ZEUS) [9] that we have used extensively for solar wind studies. The calculations we present were initiated at an inner boundary of 0.14 AU, which lies well outside the critical point where the solar wind becomes supersonic. Speed, density, and temperature were first held steady at the inner boundary until a stationary, supersonic flow with a speed of $760 \mathrm{~km} \mathrm{~s}^{-1}$ at $5 \mathrm{AU}$ and a density of $2.5 \mathrm{~cm}^{-3}$ at $1 \mathrm{AU}$, matching average high latitude conditions observed by Ulysses, filled the computational mesh. The computed speed and pressure at the appropriate distance were then compared to the observed plasma profiles. For each event, a number of different initiations were tried. Here we report only the results of the trials having best agreement.

The October 24 event was initiated as a squarewave density perturbation of 8 times the initial ambient density, and having a duration of 8 hours. As discussed in [2], modeling it as a density pulse creates the initial internal overpressure required to generate an over-expanding CME, which is exactly what results (Fig. 1). The duration of 8 hours was selected based on the observed duration of the event at the Sun. This is probably an overestimate, necessitated because we have found it difficult otherwise to reproduce the disturbance duration of 63 hours observed at Ulysses. Even at 8 hours, we still do not reproduce the Ulysses duration. Qualitatively, however, we do get good agreement with the overall shape of the disturbance at Ulysses. The agreement improves in Figure 1 when we stretch out the temporal scale of the simulation results (dashed line).

The November 22 event has a more complex initiation: a saw-tooth velocity pulse up to $1400 \mathrm{~km} \mathrm{~s}^{-1}$ is first introduced turning up suddenly and tailing off over 20 hours, and a bell-shaped density pulse of 8 times the ambient density is initiated at the midpoint of the velocity pulse, lasting 10 hours. We chose this initialization to generate the observed strong forward shock followed at the rear of the CME by a reverse compression wave (Fig. 2). The reverse wave does not appear if we introduce a velocity pulse alone, nor does it appear if the velocity pulse completely overlaps the density pulse and does not lead it. The physical rational for such an initial profile might be a filament eruption in which an overlying eruption drags out denser filament material below it.

We find that within the already mentioned limitations of a 1-D HD simulation, we do get reasonable qualitative agreement with the observations, suggesting that we have a basic understanding of the dynamical evolution of these CMEs. However, to reproduce the observations in any detail, we require not only more sophisticated simulations (multi-dimensional and magnetohydrodynamic), but also better observational constraints. The primary limitations are a lack of measurements of the initial density, temperature or field, as well as the line-of-sight confusion resulting from single-viewpoint observations. It would be extremely useful to coordinate in situ measurements with CME density and temperature measurements like those obtained from the Ultraviolet Coronagraph Spectrometer on board SOHO [10]. The line-of-sight issue will be addressed by the STEREO mission, which consists of two spacecraft orbiting the Sun such that they produce stereoscopic images of the corona.

\section{ACKNOWLEDGMENTS}

Work at Los Alamos was performed under the auspices of the U. S. Department of Energy with support from NASA's Ulysses program. OCS acknowledges partial support from National Space Weather Program grant ATM-0196112 and from NASA contract S-8670-E. PR gratefully acknowledges the support of NASA and the NSF.

\section{REFERENCES}

1. D. J. McComas, H. A. Elliot, J. T. Gosling et al., Geophys. Res. Lett. 29 (2002).

2. J. T. Gosling, D. J. McComas, J. L. Phillips et al., Geophys. Res. Lett. 21, 2271 (1994).

3. S. J. Bame, D. J. McComas, B. L. Barraclough et al., Astron. Astrophys. Suppl. Ser. 92, 237 (1992).

4. G. E. Brueckner, R. A. Howard, M. J. Koomen et al., Sol. Phys. 162, 357 (1995).

5. J. P. Delaboudiniere, G. E. Artzner, J. Brunaud et al., Sol. Phys. 162, 291 (1995).

6. H. O. Funsten, J. T. Gosling, P. Riley et al., J. Geophys. Res. 104, 6679 (1999).

7. P. Riley, J. T. Gosling, and V. J. Pizzo, J. Geophys. Res. 102, 14677 (1997).

8. D. Odstrcil and V. J. Pizzo, J. Geophys. Res. 104, 493 (1999).

9. J. M. Stone, D. Mihalas, and M. L. Norman, Astrophys. J. Suppl. Ser. 80, 819 (1992).

10. A. Ciaravella, J. C. Raymond, F. Reale et al., Astrophysical Journal 557, 351 (2001). 